became deficient or, as seems more likely, the suppression of circulating antibody was a consequence of the increasing tumour mass. The Chester Beatty team have now confirmed that cytotoxic autoantibodies can be provoked in melanoma patients lacking them, by immunization with the irradiated cells of their own excised tumours. The immunization procedure, employing multiple inoculation sites, apparently bypasses the regional immunological unresponsiveness that is already present.

It is disappointing but not really surprising that immunization had no detectable effect on the clinical course of the disease. Circulating cytotoxic antibodies would be expected to be effective only against cells to which there was good physical access-such as those in the bloodstream, which might otherwise cause distant metastases. Moreover, the artificially provoked serum antibody persisted for only one to two weeks, with a tendency toward the longer time in patients with little residual disease. Perhaps antibody was absorbed by the residual tumour, which was itself ineffective in stimulating further antibody formation because of regional immunological insufficiency.

It may now be asked how can immunotherapy provide an effective anti-cancer weapon. Doubtless the rejection of solid tumours will be effected only by specifically sensitized lymphocytes and when the circumstances favour the host-such as minimal tumour mass together with a maximal immunological response. The latter can apparently be influenced by artificial immunization, and there is promise that an effective supplementary weapon will be provided by the extracorporeal culture of immune lymphocytes now going on:at Roswell Park Memorial Institute of cancer research. Methods, whether surgical or chemical, of radical reduction of melanoma mass with minimal interference with immunological efficiency should be energetically pursued.

\section{Recurrent Infection and Deficiency of Complement}

In laboratory investigations the various components of haemolytic complement have been shown to possess several activities of possible value in the defence against invasion by micro-organisms. These activities include lysis,-the generation of a factor chemotactic for polymorphonuclear cells ${ }^{1}$, and of another factor rendering micro-organisms susceptible to phagocytosis by these same cells. ${ }^{2}$ Despite these activities the actual participation of complement in antimicrobial defence has been extremely difficult to prove. This is partly because of the complexity of complement itself and partly because the recognition of deficiency of the individual components, now known to number at least nine if subcomponents are excluded, has only recently become possible. The simultaneous publication of two cases ${ }^{3}{ }^{4}$ of recurrent infection attributed to two distinct deficiencies of complement may serve to direct attention to the important protective role of complement components.

The first case was a young man with Klinefelter's syndrome

1 Ward, P. A., Cochrane, C. G. and Müller-Eberhard, H. G., Immunology 1966, 11, 141 .

2 Johnston, R. B., jun., Klempere, M. R., Alper, C. A., and Rosen, F. S., Fournal of Experimental Medicine, 1969, 129, 1275.

3 Alper, C. A., Abramson, N., Johnston, R. B., jun., Jandl, J. H., and Rosen, F. S., New England fournal of Medicine, 1970, 282, 349.

4 Miller, M. E., and Nilsson, U. R., New England fournal of Medicine, 1970, 282, 354 . who had recurrent pyogenic infections necessitating admission to hospital on 28 occasions between infancy and the age of 25 . No deficiency of cellular immunity or of any of the immunoglobulins was detected, and the humoral response to tetanus toxoid was normal. But overall complement activity, as measured by haemolysis of sensitized cells, was only about one-third the $\frac{\varrho}{c}$ normal, though of the first six components only C3 was deficient. As measured immunologically it amounted to only $\stackrel{\vec{P}}{\oplus}$ some $4-8^{\prime \prime}$, of the normal level apparently because of rapid $\bar{C}$ conversion to the inactive form C3b. Since the full activity of $\frac{\bar{\sigma}}{\bar{D}}$ this patient's complement could not be restored by addition $\vec{\Phi}$ of pure C3 but only by whole serum, it was concluded that the underlying fault was the absence of a normal inhibitor of a protease which specifically inactivates $\mathrm{C} 3$ by conversion to $\overrightarrow{0}$ $\mathrm{C} 3 \mathrm{~b}$. The addition of normal serum but not of pure C3 pro- $\overrightarrow{\vec{H}}$ vides the missing inactivator of this protease.

The second patient was a baby girl 4 months old with을 severe. eczematoid dermatitis and repeated infections with Gram-negative bacteria and staphylococci. Unlike the first case there was a familial abnormality in the antimicrobial $\vec{N}$ defences, as shown by the considerable impairment of the normal ability of the serum to enhance the phagocytic activity of polymorphcnuclear cells. This defect was a functional 근 abnormality of $\mathrm{C} 5$, which could be readily made good by the $N$ addition of normal serum, purified $\mathrm{C} 5$, or normal mouse $ᄃ$ serum, but not serum from mice congenitally deficient in C5.

There can be little doubt that similar cases will soon be $\vec{\omega}$ recognized in increasing numbers and it is important that they should be, since transfusion with fresh serum is a simple and efficient method of treatment.

\section{Folate and Vitamin $B_{12}$ in Epilepsy}

Megaloblastic anaemia developing in patients who had been on anticonvulsants was described in $1954 .^{12}$ C. F. Hawkins and M. J. Meynell ${ }^{2}$ found that their first patient failed to $\frac{\sigma}{3}$ respond to vitamin $B_{12}$ but did respond well to folic acid. Later they found confirmatory evidence of this blood change $\frac{O}{3}$ in a series of epileptics in institutions, ${ }^{3}$ though frank anaemia was not common.

It has also been known for many years that some epileptics, $\stackrel{\circ}{\circ}$ children in particular, who require prolonged and sometimes $\frac{7}{0}$ heavy dosage with anticonvulsant drugs may show. a steady deterioration of mental performance. While this may start in a child whose brain is already damaged, it may also occur in $\omega$ children previously of good intelligence. It is by no means uncommon in hospitals caring for the chronic epileptic: The importance of a low serum folate in these patients was shown by J. S. Malpas and his colleagues, ${ }^{4}$ and their mental state could be improved by treatment with folic acid. ${ }^{5}$ Unfortunately this resulted in an increase in fits, and at the same time the vitamin $B_{12}$ level in the serum fell. Giving vitamin $B_{12}$ then diminished $\stackrel{\mathbb{Q}}{\varrho}$ the fits, and F. B. Gibberd confirmed ${ }^{6}$ that if folic acid and vitamin $\mathrm{B}_{12}$ were given together to these patients the fits did 8 not increase. Why anticonvulsants act in this way is not clear.

1 Badenoch, J., Proceedings of the Royal Society of Medicine, 1954, 47, 426.

2 Hawkins, C. F., and Meynell, M. J., Lancet, 1954, 2, 737.

3 Hawkins, C. F., and Meynell, M. J., Quarterly fournal of Medicine, 1958, 27, 45 .

Malpas, J. S., Spray, G. H., and Witts, L. J., British Medical fournal, 1966, 1, 955 .

Reynolds, E. H., Lancet, 1967, 1, 1086

Gibberd, F. B., British Medical fournal, 1969, 4, 281.

Baugh, C. M., and Krumdieck, C. L., Lancet, 1969, 2, 519. 